\title{
Prognostic value of reduced heart rate variability after myocardial infarction: clinical evaluation of a new analysis method
}

\author{
T R Cripps, M Malik, T G Farrell, A J Camm
}

\begin{abstract}
The relation between heart rate variability, measured from standard 24 hour electrocardiogram recordings in patients convalescent after a myocardial infarction, and the occurrence of sudden death and spontaneous, symptomatic, sustained ventricular tachycardia were assessed in a consecutive series of 177 patients admitted with acute myocardial infarction and surviving to 7 days. In addition to the analysis of heart rate variability, the occurrence of non-sustained arrhythmias on 24 hour electrocardiographic monitoring, and the results of clinical assessment, signal averaged electrocardiography and ejection fraction were analysed and were related to outcome. During a median of 16 months of follow up (range 10-30 months) there were 17 end point events (11 $(6 \cdot 2 \%)$ sudden deaths) and six (3.4\%) episodes of sustained ventricular tachycardia. An index of the width of the frequency distribution curve for the duration of individual $R R$ intervals was used to measure heart rate variability. This mean (SD) index was significantly smaller in those with end point events $(16.8(8.0))$ than in those without events $(29.0(11 \cdot 2))$. The relative risk of the occurrence of an end point event in those with a heart rate variability index $<25$ was $\mathbf{7 \cdot 0}$. Multivariate analysis showed that of all the variables examined a reduced heart rate variability index was the single most powerful predictor of end point events.

Measurement of heart rate variability by this simple, automated, operatorindependent method provided useful information on the arrhythmic propensity in patients convalescent after myocardial infarction.
\end{abstract}

Department of Cardiological Sciences, St George's Hospital Medical School, London

T R Cripps

M Malik

T G Farrell

A J Camm

Correspondence to Dr T R Cripps, Cardiac Department, John Radcliffe Hospital, Headington, Oxford OX3 9DU.

Accepted for publication 31 July 1990 pathetic activity in relation to sympathetic activity, and that this facilitates arrhythmogenesis. ${ }^{2}$

The standard deviation method for measuring heart rate variability can be laborious, because noise and artefact (from which no clinical quality long term electrocardiogram recording is completely free) can considerably influence the results. Visual checking and correction are therefore required. ${ }^{1}$

We devised a simple, arithmetic method to produce an index of heart rate variability from clinical quality long term electrocardiogram recordings without the need for rigorous checking or correction, so that the analysis can be carried out rapidly and independently of operator skill in the clinical setting. ${ }^{3}$ We have assessed the prognostic value of analysis of heart rate variability by this method, in relation to other prognostic variables including clinical assessment, signal averaged electrocardiography, measurement of ejection fraction, and non-sustained ventricular arrhythmias on long term electrocardiographic recordings, in a consecutive series of convalescent patients after infarction.

\section{Patients and methods}

PATIENTS

All patients aged $\leqslant 70$ admitted to the hospital between 1 May 1986 and 1 June 1988 as general medical emergencies having sustained an acute myocardial infarction within the previous 24 hours were seen within 48 hours of admission and were considered for inclusion in the study, which was approved by the regional ethics committee. Acute myocardial infarction was diagnosed when two or more of the following features were present: (a) chest pain suggestive of myocardial ischaemia persisting for at least 20 minutes and unrelieved by glyceryl trinitrate; (b) a sequential rise and fall in the plasma concentrations of aspartate transaminase, 2hydroxybutyrate dehydrogenase, and/or creatine kinase with a peak concentration at least twice the upper limit for our laboratory; and $(c)$ the development of new abnormal $Q$ waves or persistent ST/T changes suggestive of non- $Q$ wave myocardial infarction. Patients were ineligible if they had non-cardiac disease likely to influence mortality, important nonischaemic cardiac disease, a history of previous cardiac surgery or permanent pacemaker insertion, and if they refused or were unable to attend for follow up. Because this was a long term prognostic study, we also excluded patients who died or had cardiac surgery within 7 days of the onset of myocardial infarction - the usual time of hospital discharge. Patients transferred from other hospitals to the regional cardiothoracic unit with 
complications of acute myocardial infarction were not included, so that the population studied would be representative of patients admitted to a general hospital and surviving to discharge after myocardial infarction. Those with previous infarction were included, as were those with $Q$ and non- $Q$ wave infarction.

\section{CLINICAL ASSESSMENT}

Infarct site (anterior or inferior/posterior) and type ( $Q$ or non- $Q$ ) was determined from all electrocardiograms recorded during admission including one at discharge. A $Q$ wave was defined as an initial negative deflection of either or both (a) $40 \mathrm{~ms}$ duration and $(b)$ at least $25 \%$ of the amplitude of the succeeding $R$ wave. The Killip class on admission was noted and the patients were reviewed daily by one of us (TRC) to document the occurrence of in-hospital complications. The important in-hospital complications were cardiogenic shock (defined as a systolic blood pressure $<90 \mathrm{~mm} \mathrm{Hg}$ with evidence of inadequate cardiac output), radiographic pulmonary oedema, persistent tachycardia (heart rate $>100$ beats/minute on the third or subsequent day), post-infarction angina or reinfarction, and blood urea $>8.4 \mathrm{mmol} / \mathrm{l}$ ).

\section{LONG TERM ELECTROCARDIOGRAPHIC} MONITORING AND ANALYSIS OF RR INTERVAL

Twenty four hour electrocardiographic monitoring was carried out by conventional techniques more than 5 days after infarction (median 7 days). In no case was the recording made after an end point had occurred. A Tracker recorder (Reynolds Medical, Hertford) was used. The patients were encouraged to continue their daily activities while the recording was made, and their medication at the time of recording was noted.

Tapes were analysed for the incidence of arrhythmias and the variability of the $R R$ interval. We used a standard operator-com-

Figure 1 Frequency distribution curves for a patient who died suddenly during follow up and one with an uncomplicated course. Though the curve for the patient who died was narrower, quantification of heart rate variability by the standard deviation ( $S D R R$ ) is misleading because of the presence of artefact. The heart rate variability (HRV) index, however, faithfully represents the visual impression of a lower variability in the patient who died.

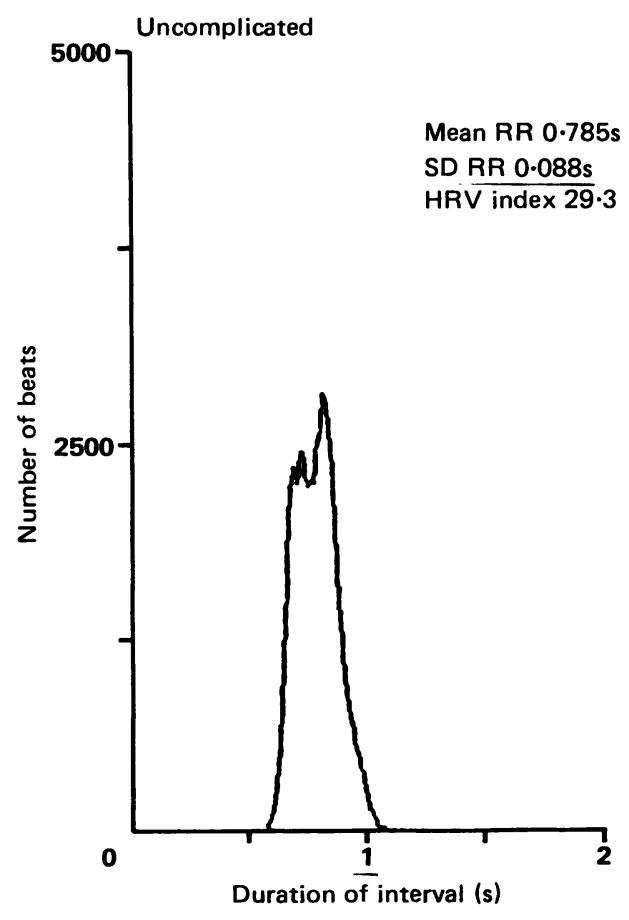

puter interactive method with a commercially available device for tape analysis (Pathfinder III, Mark II, Reynolds) to measure the frequency of extrasystoles and the occurrence of couplets, non-sustained ventricular tachycardia, and multiform ventricular extrasystoles. The analysis system identified the different shapes of the aberrant beats; the triggering level for this identification can be adjusted by the operator. In each patient we did an initial standard check to ensure that normal and extrasystolic complexes were triggering the equipment correctly. A frequency of $>10$ extrasystoles/hour was regarded as abnormal $^{4}$; no patient was given antiarrhythmic treatment on the basis of the long term electrocardiogram recording.

To measure the variability of the $R R$ interval we digitised the tapes and transferred the digital information via a serial link to a personal computer (IBM PC-AT). Each beat was tagged as normal or aberrant according to its recognition by the algorithm for tape analysis. A frequency distribution for all of the normal-normal $R R$ intervals was then generated, and the mean $R R$ interval duration, the modal $R R$ interval frequency, the standard deviation of all the normal-normal RR intervals, and the total number of recognised normal complexes were recorded (fig 1). Aberrant-normal and normal-aberrant beat intervals were excluded from analysis. Only normal-normal beat intervals were analysed.

The development of the most meaningful method for quantifying heart rate variability from such data was described elsewhere. ${ }^{3} \mathrm{We}$ found that the use of the standard deviation of RR intervals was not the most meaningful method, because, as can be seen from fig 1 , artefact can heavily weight the result. One approach to this is to check and eliminate the artefactual intervals visually. The alternative approach, which we have adopted, is to devise an arithmetic method of expressing heart rate

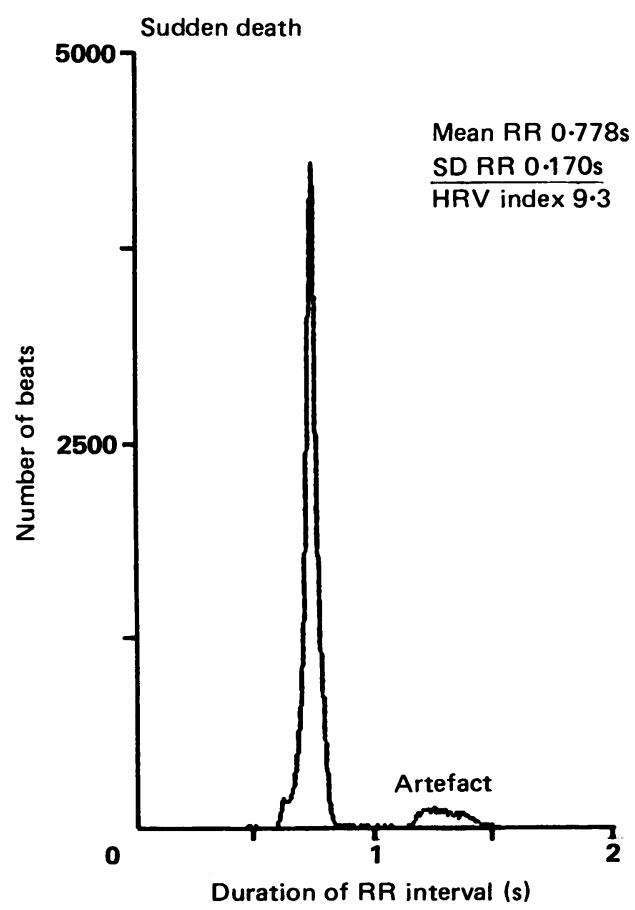


Figure 2 Triangular method for analysis of heart rate variability. The main peak of the $R R$ interval frequency distribution diagram is approximated to a triangle. Heart rate variability is quantified from the length of the base of this triangle, calculated according to simple geometrical rules.

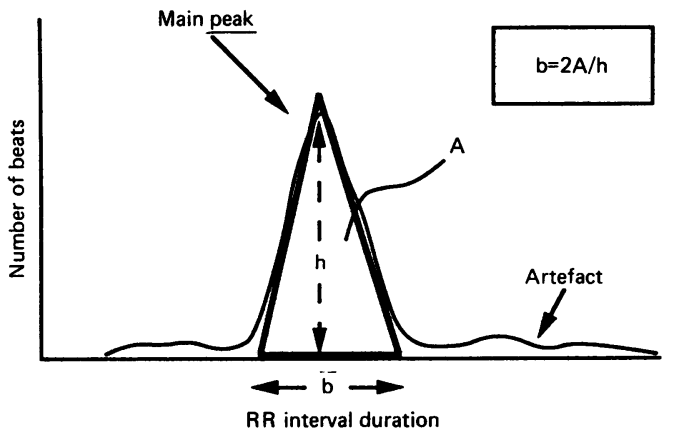

variability that is resistant to the low level of artefact found in clinical quality 24 hour tape recordings.

Of all the arithmetic methods tested ${ }^{3}$ the best for discrimination between post-infarction recordings from patients with and without arrhythmic events was the method shown in fig 2 . If the main peak on the frequency distribution diagram for computer-recognised $R R$ intervals is regarded as a triangle, its baseline width (b) (which expresses the amount of RR interval variability) can, according to simple geometrical rules, be calculated from the triangle's height (h) and area (A). The "height" is equal to the most frequently observed duration of RR interval (the modal frequency) and the "area" is (since the frequency of artefactual intervals is small compared with the frequency of the non-artefactual ones) approximately equal to the total number of computer recognised RR intervals. This "triangular index" is thus derived from a trivial calculation:

Triangular index $=$ total number

of intervals/modal RR interval frequency. The dimensions of the index are related to time, but we think that it is clearer to leave the index without units.

The triangular index tends to exaggerate the variability when the distribution of $R R$ intervals is bimodal or polymodal, but since in such cases the variability is necessarily greater than where the frequency distribution consists of a single, narrow peak, which tends to be the case in the complicated cases (fig 1) this effect tends if anything to increase the ability of the method to distinguish the high risk cases.

HIGH GAIN, SIGNAL AVERAGED

ELECTROCARDIOGRAM

Signal averaged electrocardiography was carried out with a commercially available high resolution electrocardiograph (Arrhythmia Research Technology, Model 1200 EPX) at a median of five days after infarction. The principles and operation of this device were reported elsewhere. ${ }^{5}$ The high-pass filter was set at 25 $\mathrm{Hz}$ and $100-200$ beats were averaged to achieve a final noise level of $<0.8 \mu \mathrm{V}$. We defined the presence of late potentials by Simson's criteria5: a prolonged filtered QRS alone was not regarded as a positive criterion if the QRS duration measured from the standard electrocardiogram was $>120 \mathrm{~ms}$. In all cases, the recording was performed at the bedside and completed within five minutes. No patient was given antiarrhythmic treatment on the basis of the signal averaged electrocardiogram results.
EJECTION FRACTION MEASUREMENT

Where possible left ventricular function was assessed from global left ventricular ejection fraction in all patients within the first month after infarction. If angiography was performed, the left ventricular end systolic and end diastolic volumes were measured by planimetry from the right anterior oblique projection and the ejection fraction was calculated by the Dodge formula. If angiography was not performed the ejection fraction was measured by standard radionuclide techniques.

\section{FOLLOW UP}

All patients were followed up at regular intervals in the outpatient clinic. Careful inquiries were made of the relatives and general practitioners of non-attenders to determine whether they had died and, if so, the mode of death. The primary end point was the occurrence of an arrhythmic event outside the context of reinfarction (sudden death or spontaneous, symptomatic, sustained ventricular tachycardia more than 48 hours after the onset of infarction).

Sudden death was defined as death within an hour of the onset of symptoms. Ischaemic events included reinfarction (defined as for the initial infarction (see above) in patients in hospital) or the occurrence of persistent uncharacteristic ischaemic type pain in those dying out of hospital.

The end points were chosen because they seemed most appropriate for a study seeking to predict ventricular tachycardia, as distinct from mortality from all causes, especially reinfarction, which tests such as the signal averaged electrocardiogram and heart rate variability analysis would not be expected to predict.

Because the population was a geographically stable one and relatively small, through personal contact we were able to discover the outcome in all who did not attend an outpatient appointment.

\section{STATISTICAL ANALYSIS}

We compared groups of continuous variables by the two group two tailed $t$ test and discrete variables by the $\chi^{2}$ test with Yates's correction where appropriate.

We used the following definitions: sensitivity, percentage of patients with an event who had a positive test result-that is, true positive/ true positive + false negative; specificity, percentage without an event with a negative result-that is, true negative/true negative + false positive; positive predictive accuracy, percentage of patients with a positive test result who suffered an event-that is true positive/ true positive + false positive; negative predictive accuracy, percentage with a negative test without an event-that is, true negative/true negative + false positive; efficiency, percentage with correct test result - that is, true positive + true negative/total; relative risk, risk of suffering an event in those with a positive test compared with those with a negative test-that is, positive predictive accuracy/(false positive/ total negative).

Multivariate analysis was carried out using 
Table 1 Clinical characteristics of included and excluded patients

\begin{tabular}{|c|c|c|c|}
\hline & Tested & Not tested & $p$ \\
\hline Number & 177 & & - \\
\hline Age $(y r)$ & $56(9)$ & $57 \quad(9)$ & NS \\
\hline Sex (\% female) & 19 & & NS \\
\hline Infarct site ( $\%$ anterior $)$ & 51 & 47 & NS \\
\hline Q wave $(\%)$ & 66 & 63 & NS \\
\hline Previous AMI (\%) & 11 & 22 & $<0.04$ \\
\hline Killip class > III $(\%)$ & 5 & 15 & $<0.02$ \\
\hline $\operatorname{IHC}(\%)$ & & & \\
\hline Ejection fraction (\%) (SD) & $54(14)$ & $50(21)$ & NS \\
\hline Death rate $(\%)$ & & 21 & $<0.002$ \\
\hline
\end{tabular}

AMI, acute myocardial infarction; IHC, occurrence of inhospital complications.

Table 2 Results (mean (SD)) of 24 hour electrocardiographic monitoring in patients with events and without

\begin{tabular}{lccl}
\hline & $\begin{array}{l}\text { Sudden death } \\
\text { and VT }\end{array}$ & No events & $p$ \\
\hline Mean RR interval (s) & $0.71(0.09)$ & $0.87(0.15)$ & $<0.0001$ \\
Standard deviation RR & $0.12(0.09)$ & $0 \cdot 12(0.05)$ & NS \\
HRV index & $16.8(8.0)$ & $29(11.2)$ & $<0.0001$ \\
Extrasystolic frequency $(/ \mathrm{h})$ & $13.2(22.5)$ & $7.3(25.7)$ & NS \\
\hline
\end{tabular}

HRV index, heart rate variability index; standard deviation $R R$, heart rate variability by the standard deviation method; VT, ventricular tachycardia.

the Cox proportional hazards model and the PHGLM procedure in the SASS mainframe program. Throughout the study probability values of $<0.05$ were regarded as statistically significant.

\section{Results}

PATIENTS

During the period of the study 230 patients were admitted to the hospital with myocardial infarction and were eligible for entry to the study according to the exclusions listed in the methods section. Four were excluded because of life threatening non-cardiac disease; two because of important non-ischaemic cardiac disease; eight because of prior cardiac surgery, two because they had permanent pacemakers, and two who refused or were unable to attend follow up.

Of the remaining patients, the tapes of six were not suitable for analysis of heart rate variability because of atrial fibrillation; in 11 technical problems prevented analysis (three completely blank, five with barely discernible $R$ waves, and three destroyed by the tape transport mechanism), and in 36 there was no tape available. There were thus 177 patients whose tapes underwent analysis. In no case did we reject a tape of a quality acceptable for clinical arrhythmia analysis for heart rate variability analysis.

There was no significant difference (table 1 )

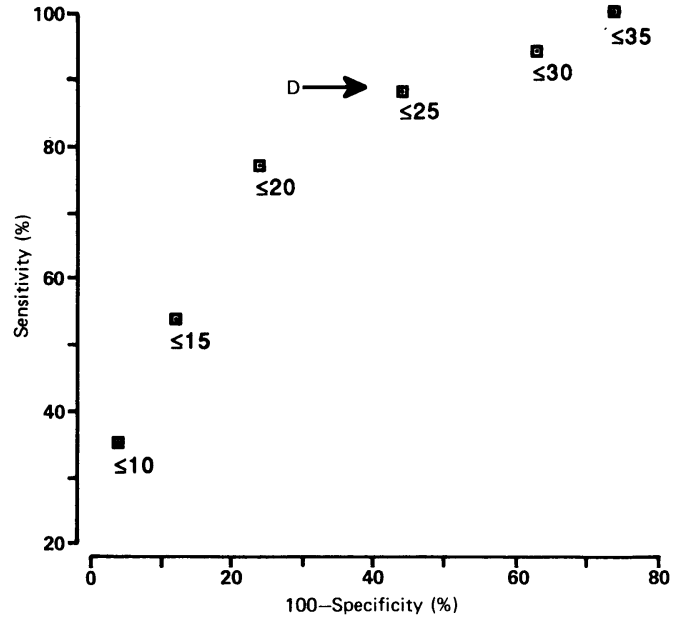

Figure 3 Receiver-operator characteristic curve (ROC curve) for heart rate variability index. The division between normal and abnormal values is indicated (D).

between eligible patients who did and did not undergo heart rate variability analysis in terms of age, sex, infarct site or type, the occurrence of important in-hospital complications, or ejection fraction. There was a slightly higher incidence of previous infarction, Killip class $>$ II, and death in those not tested: this was because several patients in whom the initial recording was not done or was not satisfactory could not undergo a repeat recording because they died soon after discharge. The clinical characteristics of the study population (table 1) show that it was comparable with other reported populations of patients surviving to discharge after acute myocardial infarction.

\section{FOLLOW UP}

Follow up of the study group was complete, with none lost, and continued for a median of 16 months (range 10-30 months). During this time, 17 patients $(9.6 \%)$ experienced end point events-11 sudden deaths $(6 \cdot 2 \%)$ and six $(3.4 \%)$ spontaneous symptomatic sustained ventricular tachycardias. There were two other deaths, one in a patient admitted with reinfarction who died of progressive cardiogenic shock culminating in electromechanical dissociation and one with atrial fibrillation who died in pulmonary oedema.

\section{LONG TERM ELECTROCARDIOGRAM RECORDINGS}

Table 2 shows the results of the long term electrocardiogram analysis, both for frequency of extrasystoles and heart rate variability. The mean RR interval was shorter. (heart rate higher) in those with events than in those without events, and the heart rate variability

Table 3 Value of 24 hour tape variables in predicting sudden death and ventricular tachycardia

\begin{tabular}{lllllll}
\hline & Sens $(\%)$ & Spec $(\%)$ & PPA $(\%)$ & NPA $(\%)$ & Eff (\%) & Relative risk \\
\hline HRV index $<25$ & 87 & 56 & 15 & 98 & 58 & $7 \cdot 0$ \\
Standard deviation RR $<50 \mathrm{~ms}$ & 67 & 63 & 14 & 95 & 63 & $3 \cdot 1$ \\
Frequency of extrasystoles $>10 / \mathrm{h}$ & 40 & 85 & 20 & 94 & 81 & $3 \cdot 3$ \\
Multiform extrasystoles & 59 & 66 & 15 & 94 & 65 & 2.5 \\
Non-sustained VT & 29 & 91 & 21 & 92 & 85 & 3.5
\end{tabular}

HRV index, heart rate variability index; Eff, efficiency; NPA, negative predictive accuracy; PPA, positive predictive accuracy; Sens, sensitivity; Spec, specificity; standard deviation RR, heart rate variability by the standard deviation method, VT, ventricular tachycardia. 
Table 4 Relation between heart rate variability index and nature of end point event

\begin{tabular}{|c|c|c|c|c|c|c|}
\hline \multirow{3}{*}{$\begin{array}{l}\text { Arrhythmic events } \\
\text { Ischaemic events }\end{array}$} & \multirow{3}{*}{$\begin{array}{c}n \\
17 \\
13 \\
\text { Sens }\end{array}$} & \multicolumn{2}{|c|}{ Events } & \multicolumn{2}{|c|}{ No events } & \multirow{2}{*}{$\begin{array}{l}p \\
0.0001 \\
\text { NS }\end{array}$} \\
\hline & & & $\begin{array}{r}(8 \cdot 0) \\
(15 \cdot 4)\end{array}$ & & $\begin{array}{l}(11 \cdot 2) \\
(11 \cdot 2)\end{array}$ & \\
\hline & & Spec & $P P A$ & $N P A$ & Eff & Relative risk \\
\hline $\begin{array}{l}\text { Arrhythmic events } \\
\text { Ischaemic events }\end{array}$ & $\begin{array}{l}87 \\
38\end{array}$ & $\begin{array}{l}56 \\
51\end{array}$ & $\begin{array}{r}15 \\
6\end{array}$ & $\begin{array}{l}98 \\
91\end{array}$ & $\begin{array}{l}58 \\
50\end{array}$ & $\begin{array}{l}7 \cdot 0 \\
0 \cdot 7\end{array}$ \\
\hline
\end{tabular}

See footnote to table 3 for abbreviations.

index was lower in those with events. There was no significant difference between the two groups, however, in terms of the mean extrasystolic frequency or the standard deviation of RR interval durations. There was no correlation between the results of analysis of heart rate variability and mortality from all causes.

The mean number of recognised normalnormal RR intervals analysed was 98581 ; in no case were fewer than 75000 intervals analysed. No patient had an aberrant beat count of $>500$ in 24 hours, and so it is clear that nearly all recognised intervals were normal-normal intervals.

Table 3 shows the results of dichotomising the 24 hour tape data into positive and negative test results. The point chosen for dichotomy was at the previously suggested level of 10 extrasystoles per hour ${ }^{4}$ and at $50 \mathrm{~ms}$ for the standard deviation of RR intervals. ${ }^{1}$ For the heart rate variability index we selected the point of optimum trade-off between sensitivity and specificity (fig 3). The optimum dichotomy points for extrasystolic frequency and standard deviation of RR intervals from our data resembled previously published values.

It is clear that, while there is an increased risk of end point events with the traditional 24 hour tape variables, namely a higher frequency of extrasystoles, multiform extrasystoles, the occurrence of non-sustained ventricular tachycardia, and a reduced standard deviation for RR intervals, the heart rate variability index is far more powerful as a predictor of primary end point events with a relative risk of event occurrence of 7.0 in those with a heart rate variability index $<25$.

Table 4 shows the prediction of arrhythmic and ischaemic events by the heart rate variability index. It seems that a reduced heart rate variability index tends to be associated, as would be expected, primarily with arrhythmic rather than ischaemic events.

OTHER PROGNOSTIC VARIABLES

Table 5 shows the results of the other prognostic tests in relation to the occurrence of end points. In 11 patients the result of the signal averaged electrocardiogram was not available, either because of bundle branch block or technical problems and in 36 the ejection fraction data were not available for administrative reasons. Table 5 shows that, unlike the other prognostic variables, reduced heart rate variability is powerfully associated with the occurrence of end point events.

\section{MULTIVARIATE ANALYSIS}

The results of multivariate analysis showed that though several of the known prognostic variables tested were individually associated with the occurrence of end point events, the single most powerful prognostic variable was the presence of a reduced heart rate variability index ( $\beta$ coefficient $-0 \cdot 159$; adjusted $\chi^{2} 10 \cdot 2$, $\mathrm{p}<0.0014)$. A reduced heart rate variability index of $<25$ provided independent prognostic information above that provided by age and mean heart rate, and the use or avoidance of $\beta$ blockers.

\section{Discussion}

This study had two aims: firstly, to apply a novel, simplified, wholly automated method for the analysis of the variability of heart rate over a 24 hour period ${ }^{3}$ to a large population of patients convalescent after myocardial infarction; and, secondly, to relate the prognostic significance of reduced heart rate variability to other, recognised prognostic variables after infarction.

Our results showed that the heart rate variability index we used gave clinically meaningful information. The relative risk of sudden death or the development of symptomatic, sustained ventricular tachycardia during follow up was seven times greater in those with an index of $<25$ than in those with an index of $\geqslant 25$ (table 3 ). This index was a much better predictor than the conventional 24 hour tape variable (an extrasystolic frequency of $>10$ per hour) both in terms of relative risk and sensitivity. As expected, however, the mean heart rate, presumably a reflection of infarct size, was also different in those with events and without. Multivariate analysis, however, showed that reduced heart rate variability did provide prognostic information independent of mean heart rate.

The other prognostic variables examined also provided useful information: the occurrence of late potentials, a reduced ejection fraction, and clinical evidence of major infarction were all associated with a relative risk of 2-4 of the occurrence of end point events

Table 5 Comparison of value of heart rate variability index with other prognostic tests for the prediction of sudden death and ventricular tachycardia

\begin{tabular}{lllllll}
\hline Test & Sens & Spec & PPA & NPA & Eff & Relative risk \\
\hline Heart rate variability index & 87 & 56 & 15 & 98 & 58 & $7 \cdot 0$ \\
Presence of late potentials & 50 & 80 & 19 & 95 & 78 & $3 \cdot 5$ \\
Admission Killip class II & 18 & 96 & 33 & 92 & 89 & $4 \cdot 0$ \\
In-hospital complications & 65 & 74 & 21 & 95 & 73 & $4 \cdot 3$ \\
Ejection fraction 40\% & 22 & 89 & 10 & 96 & 86 & $2 \cdot 2$ \\
\hline
\end{tabular}

See footnote to table 3 for abbreviations. 
(table 5). Each individual test, however, seemed to be less powerful than reduced heart rate variability in predicting the occurrence of sudden death and spontaneous, sustained ventricular tachycardia.

Only a small number of end points occurred in our relatively small population, and so it is not possible to extrapolate the results of this study to other populations with any confidence. Calculated confidence intervals are 74 to $100 \%$ for sensitivity, 48 to $64 \%$ for specificity, and 0 to $55 \%$ for predictive accuracy. However, our results are in accord with the other large scale study of heart rate variability analysis in postinfarction patients. ${ }^{1}$

Our study was retrospective in the sense that the division between a normal and an abnormal index of heart rate variability was selected to give the best discrimination between patients with and patients without arrhythmic events. The prognostic value of the index was thus optimised, and a proper comparison with the other prognostic variables must await further prognostic studies. Because the technique we describe is simple and rapid to perform, such studies are entirely feasible. Also, in the multivariate analysis, heart rate variability was treated as a continuous variable, and so the results were not biased by a favourable division into normal and abnormal.

Our result depended, of course, on the beat recognition method used by the long term electrocardiogram analysis system. Different systems should be studied too.

In this study we did not further investigate the mechanism of the relation between reduced heart rate variability and the occurrence of arrhythmic events. It seems that reduced heart rate variability reflects a disturbance of sympathovagal interaction towards a decrease in parasympathetic activity. ${ }^{6}$ Such an imbalance is thought to facilitate the emergence of ventricular tachycardia. ${ }^{7}$ Increased sympathetic activity is of course related to impaired left ventricular function, but multivariate analysis showed that in this population a reduction in heart rate variability did provide prognostic information above that obtainable from recognised indices of left ventricular impairment, including the ejection fraction. In other words, not all of those with large infarcts were at increased risk of arrhythmic events; the presence of reduced heart rate variability helped to focus more accurately upon those who were.

Efficient risk stratification after myocardial infarction is desirable, to reassure the low risk group, to spare this group further investigation and treatment, and to focus attention upon the high risk patients, on whom novel treatments can be tested effectively. It seems that analysis of heart rate variability can add to the accuracy of non-invasive prognostic stratification after acute infarction; the method of analysis we have described has the advantage of being computationally simple, rapid, repeatable, and operator independent.

1 Kleiger RE, Miller JP, Bigger JT Jr, Moss AJ, and the Multicenter Post-infarction Research Group. Decreased heart rate variability and its association with increased mortality after acute myocardial infarction. Am J Cardiol 1987;59:256-62

2 Lombardi F, Sandrone G, Pernpruner S, et al. Heart rate variability as an index of sympathovagal interaction after acute myocardial infarction. Am J Cardiol 1987;60: 1239-44.

3 Malik M, Farrell T, Cripps T, Camm AJ. Heart rate variability in relation to prognosis after myocardial infarction. Selection of optimal processing techniques. Eur Heart J 1989;10:1060-74.

4 Bigger JT Jr. Risk stratification after myocardial infarction. Z Kardiol 1985;74 (suppl 6):147-51.

5 Simson MB. Use of signals in the terminal QRS complex to identify patients with ventricular tachycardia after myocardial infarction. Circulation 1981;64:235-42.

6 Pagani M, Lombardi F, Guzzetti S, et al. Power spectral analysis of heart rate and arterial pressure variabilities as a marker of sympathovagal interaction in man and conscious dog. Circ Res 1986;59:178-93.

7 Corr PB, Yamada KA, Witkowski FX. Mechanisms controlling cardiac autonomic function and their relationships to arrhythmogenesis. In: Fozzard HA, Haber E, Jenning RB, Katz AM, Morgan HE, eds. The heart and cardiovascular system. New York: Raven Press, 1986: 1343-404. 